\title{
Antifungal Activity of New Bacterial Biocontrol Agents against Diplocarpon rosae Causing Black Spot Disease of Rose
}

\author{
Shalini $^{1}$, M. Jayasekhar ${ }^{2}$, K. G. Sabarinathan ${ }^{1}$, R. Akila ${ }^{1}$ and R. Kannan ${ }^{1}$ \\ ${ }^{1}$ Department of Plant Pathology, Agrl. College \& Res. Institute, \\ TNAU, Killikulam-628252, India \\ ${ }^{2}$ Agricultural Research Station, Tamil Nadu Agricultural University, \\ Thirupathisaram-629901, India \\ *Corresponding author
}

A B S T R A C T

K e y w o r d s
Rose, Black spot,
Biological control,
Brevibacillus sp.,
Lysinibacillus
fusiformis

The phylloplane and rhizosphere microbes of rose $c v$. Edward was isolated and nine bacteria were selected to observe their antagonistic efficacy against Diplocarpon rosae causing blackspot disease in rose. The per cent inhibition of mycelial growth of the fungi by bacterial isolates was observed. The bacterial isolates PB1 and PB2 recorded 100 per cent inhibition followed by SB1, PB2 and SB2 isolates. After molecular characterization the isolates PB1 and PB3 were found to be Pseudomonas aeruginosa and it causes life threatening infections in human beings however, the isolates SB1, SB2 and PB2 were identified as Bacillus subtilis, Brevibacillus sp., Lysinibacillus fusiformis respectively. In the field experiments the native isolate Brevibacillus sp. (SB2) was highly effective in reducing the leaf spot disease incidence by 42.94 and 33.39 per cent in Kashmir rose and Edward rose varieties with C:B ratio of 1:2.69 and 1:2.82 respectively followed by L.fusiformis (PB2) with 38.26 and 31.07 per cent reduction in disease incidence by reducing the defoliation with a $\mathrm{C}: \mathrm{B}$ ratio of $1: 2.60$ and 1:2.59. This study indicated that the new bacterial isolates isolated from rhizosphere (Brevibacillus $s p$.) and phylloplane (L.fusiformis) of rose $c v$. Edward have the potential to produce antifungal compounds which can be used to control the black leaf spot disease of rose caused by D.rosae.

\section{Introduction}

Roses are one of the most popular and economically important ornamental flowers, grown worldwide. Form, colour, texture and fragrance of flowers are the various positive attributes for the versatile use of roses in landscaping. The flower quality gets affected due to their susceptibility to diseases. Black spot disease of rose caused by Diplocarponrosae Wolf (Marssoninarosae, asexual stage) is the most destructive and widespread disease of rose worldwide (Bhaskaran and Ranganathan, 1974; Nelson, 2012; Bowen and Roark, 2001; Wenefrida and Spencer, 1993). 
Black coloured circular spots with feathery margins are produced on the upper surface of leaf. The spots are surrounded with yellow halo. The black lesions gradually increase in size and the whole leaf becomes yellow and defoliates. Due to its aesthetic value, the rose plants are used for landscaping but due to the black lesions, yellowing and defoliation of leaves, the plants become unattractive (Debener et al., 1998). Except the driest regions, this disease is found worldwide in other rose growing regions. The infection of D. rosae leads to defoliation and debilitation of the plants (Gachomo et al., 2010). Since, the use of chemical fungicides has been restricted due to their environmental and human health hazards, beneficial microbes are being experimented exclusively for the control of plant diseases. Various rhizobacteria and endophytic bacteria have been identified as biocontrol agents against plant diseases as well as most of them promote plant growth and induce disease resistance in plants. The present study aimed at evaluating the antagonistic effect of rhizobacteria and endophytic bacteria isolated from rose $c v$. Edward, against D.rosae.

\section{Materials and Methods}

\section{Sampling and bacterial isolation}

\section{Plant sample collection}

Rose $c v$. Edward grown in farmers field at Kozhikode Pottai, Thovalai was selected. Healthy and disease free leaves were collected using sterile scissors and forceps, placed in sterile polyethylene bags and kept in ice box. In the laboratory, the leaves were washed under running tap water and shade dried.

\section{Isolation of phylloplane bacteria}

Endophytic bacteria from adaxial and abaxial surface of leaf were isolated by leaf impression method from internal leaf tissue by serial dilution plating method.

\section{Serial dilution plating method}

Surface sterilization was done by washing the leaves in $0.1 \%$ mercuric chloride for $30 \mathrm{sec}$ followed by sterile water three times. Surface sterilized leaves were cut into small segments using sterile blade and macerated in $5 \mathrm{ml}$ of $12.5 \mathrm{mM}$ potassium phosphate buffer of $\mathrm{pH}$ 7.2 using sterile pestle and mortar.

The macerated tissue extracts were serially diluted in potassium phosphate buffer $\left(10^{-1}\right.$ to $\left.10^{-6}\right), 100 \mu \mathrm{l}$ of diluted samples were placed on Nutrient Agar medium and incubated at $28^{0} \mathrm{C}$ for $72 \mathrm{hrs}$. Morphologically different bacterial colonies were streaked separately and streaking was repeated until pure colonies were obtained.

\section{Leaf impression method}

A single leaf was taken and its imprint was made on Nutrient Agar plate by smoothly pressing it on agar surface using a sterile glass rod. Imprints of both abaxial and adaxial leaf surface were made. The plates were incubated at $28^{\circ} \mathrm{C}$ for $72 \mathrm{hrs}$. Bacterial colonies were observed on the leaf imprints.

\section{Isolation of rhizosperic bacteria from soil sample}

Soil samples from the rhizosphere region of the rose plants were collected in sterile polyethylene bags and placed in ice box. After reaching laboratory, $10 \mathrm{~g}$ of soil sample was put into $250 \mathrm{ml}$ conical flask containing $90 \mathrm{ml}$ of sterile water and allowed to settle down. Then the suspension was serially diluted in sterile water from $10^{-1}$ to $10^{-7} .100$ $\mu \mathrm{l}$ of diluted suspension samples from $10^{-5}$ to $10^{-7}$ were cultured on Nutrient agar medium at $28^{\circ} \mathrm{C}$ for $72 \mathrm{hrs}$. The morphologically different bacterial isolates were subcultured 
using streak plate method to obtain a pure colony.

\section{Dual plate method}

The isolated bacterial cultures were tested against the pathogen by dual plate technique. PDA medium was freshly prepared and autoclaved. Twenty $\mathrm{ml}$ of autoclaved medium was poured into sterilized Petri plates and allowed to solidify. The bacterial isolates were then streaked on the solidified medium at a distance of $3 \mathrm{~cm}$ from the rim of the plate. Using sterile cork borer nine $\mathrm{mm}$ diameter fungal disc of $D$. rosae was cut from old culture and placed on the other side of Petri plate. Three replications were maintained for each treatment. The inoculated plates were incubated at $25 \pm 1{ }^{\circ} \mathrm{C}$ for seven days. The diameter of the mycelial growth was documented and the per cent inhibition was calculated. The plate inoculated only with fungal disc was used as control.

\section{Molecular characterization of isolated bacteria}

Molecular characterization was done using 16S rDNA gene sequence analysis with isolates SB1, SB2, PB1, PB2 and PB3 which showed maximum inhibitory effect against the pathogen. The total genomic DNA was isolated from the bacterial isolates by the CTAB method (Gomes et al., 2000). 27F and $1115 \mathrm{r}$ primers were used for PCR amplification of the $16 \mathrm{~S}$ rDNA gene, which was performed in $25 \mu \mathrm{l}$ reaction using the following conditions: initial denaturation at $94^{\circ} \mathrm{C}$ for $5 \mathrm{~min}$ followed by 35 cycles of denaturation at $94^{\circ} \mathrm{C}$ for $30 \mathrm{sec}$, annealing at $50^{\circ} \mathrm{C}$ for $30 \mathrm{sec}$, extension at $72^{\circ} \mathrm{C}$ for $2 \mathrm{~min}$ and a final extension at $72^{\circ} \mathrm{C}$ for $7 \mathrm{~min}$ on Eppendorf master cycler gradient PCR machine. The amplified product was purified using PCR purification Kit and sequenced by Eurofins genomics India Pvt. Ltd., Bangalore.
Similarity searches of the sequences were carried out using the BLAST function of GenBank.

\section{Evaluation of effective bacterial isolates against $D$. rosae under in vivo condition}

The experiment was conducted in farmer's rose field at Kozhikode Pottai, Thovalaitaluk, Kanyakumari District, Tamil Nadu. According to the guidelines given in crop production guide (CPG), the field was maintained with proper spacing of $2 \times 1 \mathrm{~m}$, proper weed management, irrigation and fertilizer application.

The effective bacterial isolates were evaluated under in vivo condition using Randomized Block Design (RBD) by comparing with three recommended chemical fungicides (CPG) and a water spray as control. All the treatments were applied on two different rose varieties viz., Scented Rose and Kashmir Rose. At an interval of 15 days after two sprayings, the observation was taken. The percentage of disease severity before spraying and after second spraying was recorded. Effect of each treatment was evaluated by analysing the disease reduction percentage and defoliation percentage. The defoliation percentage was calculated by recording the number of leaves present on a particular tagged stem of plants before spraying and at 15 days interval after second spraying. The flower count per plant was also documented for all treatments after second spraying and the cost benefit ratio was calculated for individual treatments.

\section{Results and Discussion}

Seven different bacteria were isolated from phylloplane and rhizospheric soil of rose plants. The standard bio control agent Pseudomonas fluorescens maintained in Department of Plant Pathology, Agricultural College and Research Institute, TNAU, 
Killikulam was also tested against the pathogen D. rosae (Fig. 1).

Effect of phylloplane and rhizosphere bacteria on the mycelial growth of $D$. rosae in vitro

The isolated bacteria and the standard bio control agent Pseudomonas fluorescens were examined by dual plate method against $D$. rosae. Among these bacteria, $\mathrm{PB} 1$ and $\mathrm{PB} 3$ completely inhibited the mycelial growth of the pathogen and showed 100 per cent inhibition over control. SB1 showed 71.44 per cent inhibition followed by SB2and PB2 each with 66.67 and65.78 per cent inhibition over control respectively. The bacterial isolate PB5 showed the least inhibition percentage of 33.33 over control (Table 1; Fig. 2).

\section{Identification of isolated bacteria by $16 \mathrm{~S}$ rDNA sequence analysis}

Amplification of $16 \mathrm{~S}$ rDNA gene by PCR resulted in a product approximately $1.1 \mathrm{~kb}$ in size. Sequencing of the PCR product followed by BLAST searches revealed that SB1 showed $99 \%$ similarity to Bacillus subtilis strain, SB2showed $96.45 \%$ similarity to Brevibacillus sp. strain, PB1 showed 96\% similarity to Pseudomonas aeruginosa strain, PB2 showed 94\% similarity to Lysinibacillus fusiformis strain and PB3 showed 95\% similarity to Pseudomonas aeruginosa strain deposited in GenBank.

After molecular characterization of the isolated bacteria, the effective isolates PB1 and PB3 were found to be Pseudomonas aeruginosa. $P$. aeruginosa causes life threatening infections in human beings (Kunert et al., 2007; Bordi and de Bentzmann, 2011). The Infectious Diseases Society of America has listed this bacteria as most dangerous human pathogen (Talbot et al., 2006). These pathogens show resistance to antibiotics, therefore drugs for controlling Pseudomonas aeruginosa are limited (Endimiani et al., 2006). Since it was a human pathogen, other isolates SB1 (Bacillus subtilis), SB2 (Brevibacillus sp.), PB2 (Lysinibacillus fusiformis) were further studied under in vivo condition.

\section{Evaluation of effective bacterial isolates} against $D$. rosaeunder in vivo condition

The effective bacterial isolates under in vitro condition and P.fluorescens were tested on the incidence of rose black spot under field condition on two different varieties viz., Kashmir Rose and Scented Rose. The fungicides Carbendazim, Hexaconazole, Tebuconazole+ Trifloxystrobin and water spray were used as control. Table 2 showed the effect of different treatments on the variety Kashmir Rose. The table revealed that the plants treated with the combination fungicide Tebuconazole+ Trifloxystrobin showed highest disease reduction percentage viz., 43.95 per cent followed by Hexaconazole (43.45), SB2- Brevibacillus sp.(42.94 per cent) andPB2-Lysinibacillus fusiformis (38.26 per cent). The least disease reduction percentage was observed in the plants treated with Pseudomonas fluorescens (26.72 per cent). The table 3 showed the defoliation percentage of the plants before spraying and at 15, 30, 45 and 60 days after second spraying. The defoliation percentage was lowest in plants treated with the combination fungicide Tebuconazole+ Trifloxystrobin (2.64 per cent) followed by Hexaconazole (5.42 per cent) and SB2-Brevibacillus sp. (7.25 per cent).The defoliation percentage was highest in plants treated with water spray (45.56 per cent). The flower yield was observed to be increased in plants sprayed with the treatments which were effective in reducing the black spot disease. The cost benefit ratio between the increased yield due to application of various treatments and the 
loss due to spray schedule was calculated. The table 4 showed that application of SB2Brevibacillus sp. was economical with cost benefit ratio of 1:2.69 followed by Hexaconazole (1:2.68) and PB2Lysinibacillus fusiformis (1:2.60).

The table 5 showed the effect of different treatments on the variety Scented Rose. The table revealed that the plants treated with the combination fungicide Tebuconazole+ Trifloxystrobin showed highest disease reduction percentage viz., 54.35 per cent followed by Hexaconazole (49.99), SB2Brevibacillus sp. (33.39 per cent) andPB2Lysinibacillus fusiformis(31.07 per cent).The least disease reduction percentage was observed in the plants treated with Carbendazim (22.81 per cent). The table 6 showed the defoliation percentage of the plants before spraying and at 15, 30, 45 and 60 days after second spraying. The defoliation percentage was lowest in plants treated with the combination fungicide Tebuconazole+ Trifloxystrobin (6.74 per cent) followed by SB2-Brevibacillus sp. (8.06 per cent) and PB2-Lysinibacillusfusiformis (8.25 per cent). The defoliation percentage was highest in plants treated with water spray (41.33 per cent). The table 7 showed that application of Hexaconazole was economical with cost benefit ratio of 1:2.87 followed by SB2Brevibacillus sp. (1:2.82) and PB2-L. fusiformis(1:2.59).

Among all the treatments evaluated, the native endophytic bacteria isolated from soilBrevibacillus sp., the native endophytic bacteria isolated from phylloplane regionLysinibacillus fusiformis and the fungicides Tebuconazole 50 per cent + Trifloxystrobin 25 per cent and Hexaconazole 5 per cent EC were highly effective in reducing the disease incidence in both the rose varieties.

Table.1 Antifungal activity of endophytic bacteria against Diplocarpon rosae

\begin{tabular}{|c|l|c|c|}
\hline Treatment & $\begin{array}{c}\text { Bio control } \\
\text { agents }\end{array}$ & $\begin{array}{c}\text { *Mycelial } \\
\text { growth }(\mathbf{c m})\end{array}$ & $\begin{array}{c}\text { Per cent inhibition } \\
\text { over control (\%) }\end{array}$ \\
\hline $\mathbf{T}_{\mathbf{1}}$ & SB1 & 2.57 & $\begin{array}{c}71.44 \\
(58.22)^{\mathrm{b}}\end{array}$ \\
\hline $\mathbf{T}_{\mathbf{2}}$ & SB2 & 3.00 & $\begin{array}{c}66.67 \\
(54.76)^{\mathrm{c}}\end{array}$ \\
\hline $\mathbf{T}_{\mathbf{3}}$ & PB1 & 0.00 & $\begin{array}{c}100.00 \\
(90.00)^{\mathrm{a}}\end{array}$ \\
\hline $\mathbf{T}_{\mathbf{4}}$ & PB2 & 3.08 & $\begin{array}{c}65.78 \\
(54.72)^{\mathrm{c}}\end{array}$ \\
\hline $\mathbf{T}_{\mathbf{5}}$ & PB3 & 0.00 & 100.0 \\
\hline $\mathbf{T}_{\mathbf{6}}$ & PB4 & 5.61 & $(90.00)^{\mathrm{a}}$ \\
\hline $\mathbf{T}_{\mathbf{7}}$ & PB5 & 6.00 & $\begin{array}{c}37.67 \\
(37.93)^{\mathrm{e}}\end{array}$ \\
\hline $\mathbf{T}_{\mathbf{8}}$ & P.fluorescens & 3.59 & 33.11 \\
& & & $(35.26)^{\mathrm{f}}$ \\
\hline $\mathbf{T}_{\mathbf{9}}$ & Control & 9.00 & $(51.45)^{\mathrm{d}}$ \\
\hline & & & 0.00 \\
\hline $\mathbf{C D}$ (P=0.05) & 0.06 & $(0.00)^{\mathrm{g}}$ \\
\hline
\end{tabular}


Table.2 Evaluation of effective bacterial isolates under field conditions on Kashmir Rose

\begin{tabular}{|c|c|c|c|c|c|c|c|c|c|}
\hline \multirow{2}{*}{\multicolumn{2}{|c|}{ Treatment }} & \multirow{3}{*}{$\begin{array}{c}\text { Conc. } \\
(\%)\end{array}$} & \multicolumn{5}{|c|}{ *Disease severity (\%) } & \multirow{3}{*}{$\begin{array}{c}\text { Mean } \\
\\
17.10 \\
(24.21)\end{array}$} & \multirow{3}{*}{$\begin{array}{c}\begin{array}{c}\text { Disease } \\
\text { reduction } \\
(\%)\end{array} \\
30.66^{\mathrm{e}}\end{array}$} \\
\hline & & & \multirow{2}{*}{$\begin{array}{c}\begin{array}{c}\text { Before } \\
\text { spraying }\end{array} \\
25.19 \\
(30.11)\end{array}$} & \multirow{2}{*}{$\begin{array}{c}\text { 15 } \\
\text { DAS } \\
19.56 \\
(26.23)\end{array}$} & \multirow{2}{*}{$\begin{array}{c}\text { 30 DAS } \\
15.25 \\
(22.98)\end{array}$} & \multirow{2}{*}{$\begin{array}{c}\mathbf{4 5} \\
\text { DAS } \\
13.56 \\
(21.52)\end{array}$} & \multirow{2}{*}{$\begin{array}{c}\mathbf{6 0} \\
\text { DAS } \\
11.94 \\
(20.22)\end{array}$} & & \\
\hline $\mathbf{T}_{1}$ & SB1 (Bacillus subtilis) & & & & & & & & \\
\hline $\mathbf{T}_{2}$ & SB2(Brevibacillus sp.) & $\begin{array}{c}10^{8} \\
\mathrm{cfu} / \mathrm{ml}\end{array}$ & $\begin{array}{c}25.23 \\
(30.16)\end{array}$ & $\begin{array}{c}18.25 \\
(25.29)\end{array}$ & $\begin{array}{c}11.24 \\
(19.59)\end{array}$ & $\begin{array}{c}9.23 \\
(17.70)\end{array}$ & $\begin{array}{c}6.41 \\
(14.67)\end{array}$ & $\begin{array}{l}14.07 \\
(21.48)\end{array}$ & $42.94^{\mathrm{b}}$ \\
\hline $\mathbf{T}_{\mathbf{3}}$ & $\begin{array}{l}\text { PB2 } \\
\text { (Lysinibacillusfusiformis) }\end{array}$ & $\begin{array}{c}10^{8} \\
\mathrm{cfu} / \mathrm{ml}\end{array}$ & $\begin{array}{l}25.21 \\
(30.09)\end{array}$ & $\begin{array}{l}19.05 \\
(25.92)\end{array}$ & $\begin{array}{l}12.56 \\
(20.61)\end{array}$ & $\begin{array}{c}11.05 \\
(19.40)\end{array}$ & $\begin{array}{c}8.25 \\
(16.78)\end{array}$ & $\begin{array}{l}15.22 \\
(22.56)\end{array}$ & $38.26^{\mathrm{c}}$ \\
\hline $\mathbf{T}_{4}$ & P.fluorescens & $\begin{array}{c}10^{8} \\
\mathrm{cfu} / \mathrm{ml}\end{array}$ & $\begin{array}{c}25.21 \\
(30.15)\end{array}$ & $\begin{array}{c}18.95 \\
(25.62)\end{array}$ & $\begin{array}{c}17.24 \\
(24.54)\end{array}$ & $\begin{array}{c}15.36 \\
(23.04)\end{array}$ & $\begin{array}{l}13.59 \\
(21.44)\end{array}$ & $\begin{array}{c}18.07 \\
(24.96)\end{array}$ & $26.72^{f}$ \\
\hline $\mathbf{T}_{5}$ & Carbendazim & 0.1 & $\begin{array}{l}25.20 \\
(30.15)\end{array}$ & $\begin{array}{c}19.62 \\
(26.12)\end{array}$ & $\begin{array}{c}14.23 \\
(22.18)\end{array}$ & $\begin{array}{c}12.54 \\
(20.69)\end{array}$ & $\begin{array}{c}9.24 \\
(17.71)\end{array}$ & $\begin{array}{c}16.17 \\
(23.37)\end{array}$ & $34.44^{\mathrm{d}}$ \\
\hline $\mathbf{T}_{6}$ & Hexaconazole & 0.05 & $\begin{array}{c}25.17 \\
(30.12)\end{array}$ & $\begin{array}{l}13.25 \\
(21.35)\end{array}$ & $\begin{array}{c}11.48 \\
(19.73)\end{array}$ & $\begin{array}{c}10.68 \\
(18.92)\end{array}$ & $\begin{array}{c}9.14 \\
(17.61)\end{array}$ & $\begin{array}{c}13.94 \\
(21.55)\end{array}$ & $43.45^{\mathrm{a}}$ \\
\hline $\mathbf{T}_{7}$ & $\begin{array}{l}\text { Tebuconazole+ } \\
\text { Trifloxystrobin }\end{array}$ & 0.05 & $\begin{array}{c}25.01 \\
(30.01)\end{array}$ & $\begin{array}{c}15.28 \\
(22.99)\end{array}$ & $\begin{array}{c}10.23 \\
(18.64)\end{array}$ & $\begin{array}{c}9.51 \\
(17.85)\end{array}$ & $\begin{array}{c}9.08 \\
(17.52)\end{array}$ & $\begin{array}{c}13.82 \\
(21.41)\end{array}$ & $43.95^{\mathrm{a}}$ \\
\hline $\mathbf{T}_{8}$ & Control (Water spray) & - & $\begin{array}{l}24.86 \\
(29.73)\end{array}$ & $\begin{array}{l}24.67 \\
(29.56)\end{array}$ & $\begin{array}{l}24.61 \\
(29.50)\end{array}$ & $\begin{array}{l}24.56 \\
(29.44)\end{array}$ & $\begin{array}{c}24.59 \\
(29.48)\end{array}$ & $\begin{array}{l}24.66 \\
(29.54)\end{array}$ & \\
\hline & Mean & & $\begin{array}{c}25.14 \\
(30.07)\end{array}$ & $\begin{array}{c}18.58 \\
(25.39)\end{array}$ & $\begin{array}{c}14.61 \\
(22.22)\end{array}$ & $\begin{array}{c}13.31 \\
(21.07)\end{array}$ & $\begin{array}{c}11.53 \\
(19.43)\end{array}$ & & \\
\hline & $\mathrm{CD}(\mathrm{P}=\mathbf{0 . 0 5})$ & & Treatment & $=0.010 \mathrm{D}$ & ys $=0.008$ & Treat & ent X Da & $=0.022$ & \\
\hline
\end{tabular}

DAS - Days after second spraying*Mean of three replications

The treatment means are compared using Duncan multiple range test (DMRT).

Figures in parentheses are arc sine transformed values

In a column, mean followed by a common letter ( $\mathrm{s})$ are not significantly different $(\mathrm{p}=0.05)$

Table.3 Defoliation percentage in treated Kashmir Rose plants

\begin{tabular}{|c|c|c|c|c|c|c|c|c|}
\hline \multirow{2}{*}{\multicolumn{2}{|c|}{ Treatment }} & \multicolumn{6}{|c|}{ *Average no. of leaflets per stem } & \multirow{2}{*}{$\begin{array}{c}\text { Defoliation } \\
\text { percentage } \\
(\%)\end{array}$} \\
\hline & & \multirow{2}{*}{$\begin{array}{c}\text { Before } \\
\text { spraying } \\
85.59\end{array}$} & \multirow{2}{*}{$\begin{array}{c}15 \\
\text { DAS } \\
81.68\end{array}$} & \multirow{2}{*}{$\begin{array}{c}\text { 30 } \\
\text { DAS } \\
78.25\end{array}$} & \multirow{2}{*}{$\begin{array}{c}45 \\
\text { DAS } \\
77.39\end{array}$} & \multirow{2}{*}{$\begin{array}{c}\text { 60 } \\
\text { DAS } \\
76.28\end{array}$} & \multirow{2}{*}{$\begin{array}{l}\text { Mean } \\
79.84\end{array}$} & \\
\hline $\mathbf{T}_{1}$ & SB1 (Bacillus subtilis) & & & & & & & 10.88 \\
\hline $\mathbf{T}_{2}$ & SB2(Brevibacillus sp.) & 86.63 & 83.05 & 82.65 & 81.06 & 80.35 & 82.75 & 7.25 \\
\hline $\mathbf{T}_{3}$ & $\begin{array}{l}\text { PB2 } \\
\text { (Lysinibacillusfusiformis) }\end{array}$ & 81.71 & 78.14 & 76.27 & 72.64 & 72.28 & 76.21 & 11.54 \\
\hline $\mathbf{T}_{4}$ & P.fluorescens & 83.2 & 78.29 & 75.67 & 72.29 & 69.55 & 75.80 & 16.41 \\
\hline $\mathbf{T}_{5}$ & Carbendazim & 84.21 & 80.39 & 76.27 & 72.36 & 71.29 & 76.90 & 15.34 \\
\hline $\mathbf{T}_{6}$ & Hexaconazole & 85.26 & 83.34 & 81.08 & 80.22 & 80.64 & 82.11 & 5.42 \\
\hline $\mathbf{T}_{7}$ & $\begin{array}{l}\text { Tebuconazole+ } \\
\text { Trifloxystrobin }\end{array}$ & 82.15 & 81.16 & 80.81 & 79.62 & 79.98 & 80.74 & 2.64 \\
\hline $\mathbf{T}_{8}$ & Control (Water spray) & 83.29 & 78.84 & 65.1 & 52.08 & 45.34 & 64.93 & 45.56 \\
\hline & $\mathrm{CD}(\mathrm{P}=0.05)$ & NS & 0.239 & 0.416 & 0.213 & 0.524 & & \\
\hline
\end{tabular}

DAS - Days after second spraying

*Mean of three replications 
Table.4 Cost benefit ratio of the treatments on Kashmir rose

\begin{tabular}{|l|l|c|c|c|c|c|c|}
\hline & \multicolumn{1}{|c|}{ Treatment } & $\begin{array}{c}\text { Average no. } \\
\text { of } \\
\text { flowers/plant }\end{array}$ & $\begin{array}{c}\text { Yield } \\
\text { /ha/year } \\
\text { (lakhs/ha) }\end{array}$ & $\begin{array}{c}\text { Increase } \\
\text { in yield } \\
\text { over } \\
\text { control } \\
\text { (lakhs/ha) }\end{array}$ & $\begin{array}{c}\text { Additional } \\
\text { cost of } \\
\text { treatment } \\
\text { (Rs) }\end{array}$ & $\begin{array}{c}\text { Cost of } \\
\text { additional } \\
\text { returns/ha at } \\
\text { Rs 150/kg } \\
(-\mathbf{8 0 0} \text { flowers) }\end{array}$ & $\begin{array}{c}\text { Cost } \\
\text { benefit } \\
\text { ratio }\end{array}$ \\
\hline $\mathbf{T}_{\mathbf{1}}$ & SB1 (Bacillus subtilis) & 9.39 & 7.04 & 0.37 & 7920 & 6984 & $1: 0.88$ \\
\hline $\mathbf{T}_{\mathbf{2}}$ & SB2 (Brevibacillus sp.) & 10.41 & 7.81 & 1.14 & 7920 & 21328 & $1: 2.69$ \\
\hline $\mathbf{T}_{\mathbf{3}}$ & $\begin{array}{l}\text { PB2 } \\
\text { (Lysinibacillusfusiformis) }\end{array}$ & 10.36 & 7.77 & 1.10 & 7920 & 20625 & $1: 2.60$ \\
\hline $\mathbf{T}_{\mathbf{4}}$ & P.fluorescens & 9.54 & 7.16 & 0.48 & 7000 & 9094 & $1: 1.30$ \\
\hline $\mathbf{T}_{\mathbf{5}}$ & Carbendazim & 10.24 & 7.68 & 1.01 & 8500 & 18938 & $1: 2.23$ \\
\hline $\mathbf{T}_{\mathbf{6}}$ & Hexaconazole & 10.78 & 8.09 & 1.42 & 9800 & 26290 & $1: 2.68$ \\
\hline $\mathbf{T}_{\mathbf{7}}$ & $\begin{array}{l}\text { Tebuconazole+ } \\
\text { Trifloxystrobin }\end{array}$ & 12.56 & 8.42 & 1.75 & 21000 & 32815 & $1: 1.56$ \\
\hline $\mathbf{T}_{\mathbf{8}}$ & Control (Water spray) & 8.89 & 6.67 & - & & & \\
\hline
\end{tabular}

Table.5 Evaluation of effective bacterial isolates under field conditions on scented rose

\begin{tabular}{|c|c|c|c|c|c|c|c|c|c|}
\hline \multirow{2}{*}{\multicolumn{2}{|c|}{ Treatment }} & \multirow{2}{*}{$\begin{array}{c}\text { Conc. } \\
(\%)\end{array}$} & \multicolumn{5}{|c|}{ *Disease severity (\%) } & \multirow[b]{2}{*}{ Mean } & \multirow{2}{*}{$\begin{array}{c}\text { Disease } \\
\text { reduction } \\
(\%)\end{array}$} \\
\hline & & & \multirow{2}{*}{$\begin{array}{c}\text { Before } \\
\text { spraying } \\
15.65 \\
(23.29)\end{array}$} & \multirow{2}{*}{$\begin{array}{c}\mathbf{1 5} \\
\text { DAS } \\
14.52 \\
(22.28)\end{array}$} & \multirow{2}{*}{$\begin{array}{c}30 \text { DAS } \\
11.63 \\
(19.95)\end{array}$} & \multirow{2}{*}{$\begin{array}{c}\mathbf{4 5} \\
\text { DAS } \\
9.24 \\
(17.76)\end{array}$} & \multirow{2}{*}{\begin{tabular}{|c|}
$\mathbf{6 0}$ \\
DAS \\
7.67 \\
$(16.09)$
\end{tabular}} & & \\
\hline $\mathbf{T}_{1}$ & SB1 (5Bacillus subtilis) & $\begin{array}{c}10^{8} \\
\mathrm{cfu} / \mathrm{ml}\end{array}$ & & & & & & $\begin{array}{c}11.74 \\
(19.88)\end{array}$ & $24.92^{\mathrm{e}}$ \\
\hline $\mathbf{T}_{2}$ & SB2(Brevibacillus sp.) & $\begin{array}{c}10^{8} \\
\mathrm{cfu} / \mathrm{ml}\end{array}$ & $\begin{array}{l}15.68 \\
(23.21)\end{array}$ & $\begin{array}{c}15.08 \\
(22.85)\end{array}$ & $\begin{array}{c}9.21 \\
(17.69)\end{array}$ & $\begin{array}{c}6.28 \\
(14.51)\end{array}$ & $\begin{array}{c}5.84 \\
(13.99)\end{array}$ & $\begin{array}{c}10.42 \\
(18.45)\end{array}$ & $33.39^{c}$ \\
\hline $\mathbf{T}_{3}$ & $\begin{array}{l}\text { PB2 } \\
\text { (Lysinibacillusfusiformis) }\end{array}$ & $\begin{array}{c}10^{8} \\
\mathrm{cfu} / \mathrm{ml}\end{array}$ & $\begin{array}{l}15.45 \\
(23.11)\end{array}$ & $\begin{array}{c}14.85 \\
(22.59)\end{array}$ & $\begin{array}{l}10.38 \\
(18.76)\end{array}$ & $\begin{array}{c}7.93 \\
(16.36)\end{array}$ & $\begin{array}{c}5.29 \\
(13.28)\end{array}$ & $\begin{array}{c}10.78 \\
(18.82)\end{array}$ & $31.07^{\mathrm{d}}$ \\
\hline $\mathbf{T}_{4}$ & P.fluorescens & $\begin{array}{c}10^{8} \\
\mathrm{cfu} / \mathrm{ml}\end{array}$ & $\begin{array}{l}15.36 \\
(23.07)\end{array}$ & $\begin{array}{c}14.92 \\
(22.74)\end{array}$ & $\begin{array}{c}11.58 \\
(19.79)\end{array}$ & $\begin{array}{c}9.34 \\
(17.92)\end{array}$ & $\begin{array}{c}8.20 \\
(16.68)\end{array}$ & $\begin{array}{l}11.88 \\
(20.04)\end{array}$ & $24.04^{\mathrm{e}}$ \\
\hline $\mathbf{T}_{5}$ & Carbendazim & 0.1 & $\begin{array}{l}15.28 \\
(22.99)\end{array}$ & $\begin{array}{c}13.76 \\
(21.57)\end{array}$ & $\begin{array}{c}11.63 \\
(19.95)\end{array}$ & $\begin{array}{c}10.45 \\
(18.81)\end{array}$ & $\begin{array}{c}9.24 \\
(17.71)\end{array}$ & $\begin{array}{l}12.07 \\
(20.20)\end{array}$ & $22.81^{\mathrm{f}}$ \\
\hline $\mathbf{T}_{6}$ & Hexaconazole & 0.05 & $\begin{array}{l}15.23 \\
(22.95)\end{array}$ & $\begin{array}{c}10.26 \\
(18.66)\end{array}$ & $\begin{array}{c}5.89 \\
(13.89)\end{array}$ & $\begin{array}{c}4.52 \\
(12.14)\end{array}$ & $\begin{array}{c}3.21 \\
(10.37)\end{array}$ & $\begin{array}{c}7.82 \\
(15.61)\end{array}$ & $49.99^{b}$ \\
\hline $\mathbf{T}_{7}$ & $\begin{array}{l}\text { Tebuconazole+ } \\
\text { Trifloxystrobin }\end{array}$ & 0.05 & $\begin{array}{c}15.29 \\
(22.99)\end{array}$ & $\begin{array}{c}9.51 \\
(17.88)\end{array}$ & $\begin{array}{c}5.28 \\
(13.26)\end{array}$ & $\begin{array}{c}3.48 \\
(10.63)\end{array}$ & $\begin{array}{c}2.14 \\
(8.43)\end{array}$ & $\begin{array}{c}7.14 \\
(14.64)\end{array}$ & $54.35^{\mathrm{a}}$ \\
\hline $\mathbf{T}_{8}$ & Control (Water spray) & - & $\begin{array}{l}15.37 \\
(23.07)\end{array}$ & $\begin{array}{c}15.65 \\
(23.19)\end{array}$ & $\begin{array}{c}15.69 \\
(23.22)\end{array}$ & $\begin{array}{c}15.74 \\
(23.32)\end{array}$ & $\begin{array}{c}15.73 \\
(23.36)\end{array}$ & $\begin{array}{c}15.64 \\
(23.23)\end{array}$ & \\
\hline & Mean & & $\begin{array}{l}15.41 \\
(23.09)\end{array}$ & $\begin{array}{c}13.57 \\
(21.47)\end{array}$ & $\begin{array}{l}10.16 \\
(18.31)\end{array}$ & $\begin{array}{c}8.37 \\
(16.43)\end{array}$ & $\begin{array}{c}7.17 \\
(14.99)\end{array}$ & & \\
\hline & $\mathrm{CD}(\mathrm{P}=0.05)$ & & Treatment & $=0.025$ & ays $=0.02$ & Treatm & t X Days & $=0.056$ & \\
\hline
\end{tabular}

DAS - Days after second spraying *Mean of three replications

The treatment means are compared using Duncan multiple range test (DMRT).

Figures in parentheses are arc sine transformed values

In a column, mean followed by a common letter ( $\mathrm{s}$ ) are not significantly different $(\mathrm{p}=0.05)$. 
Table.6 Defoliation percentage in treated scented rose plants

\begin{tabular}{|c|c|c|c|c|c|c|c|c|}
\hline \multirow{2}{*}{\multicolumn{2}{|c|}{ Treatment }} & \multicolumn{5}{|c|}{ * Average no. of leaves per stem } & \multirow{3}{*}{$\begin{array}{l}\text { Mean } \\
60.58\end{array}$} & \multirow{3}{*}{$\begin{array}{c}\begin{array}{c}\text { Defoliation } \\
\text { percentage } \\
(\%)\end{array} \\
12.46\end{array}$} \\
\hline & & \multirow{2}{*}{$\begin{array}{c}\text { Before } \\
\text { spraying } \\
65.25\end{array}$} & \multirow{2}{*}{$\begin{array}{c}15 \\
\text { DAS } \\
62.25\end{array}$} & \multirow{2}{*}{$\begin{array}{c}\text { 30 } \\
\text { DAS } \\
58.74\end{array}$} & \multirow{2}{*}{$\begin{array}{c}45 \\
\text { DAS } \\
58.64\end{array}$} & \multirow{2}{*}{$\begin{array}{c}\mathbf{6 0} \\
\text { DAS } \\
58.02\end{array}$} & & \\
\hline $\mathbf{T}_{1}$ & SB1 (Bacillus subtilis) & & & & & & & \\
\hline $\mathbf{T}_{2}$ & SB2 (Brevibacillus sp.) & 66.26 & 64.36 & 61.89 & 61.27 & 61.32 & 63.02 & 8.06 \\
\hline $\mathbf{T}_{3}$ & $\begin{array}{l}\text { PB2 (Lysinibacillus } \\
\text { fusiformis) }\end{array}$ & 67.31 & 64.48 & 63.64 & 63.29 & 62.18 & 64.18 & 8.25 \\
\hline $\mathbf{T}_{4}$ & P.fluorescens & 69.58 & 65.27 & 60.33 & 58.48 & 58.35 & 62.40 & 19.25 \\
\hline $\mathbf{T}_{5}$ & Carbendazim & 69.16 & 60.09 & 57.16 & 56.39 & 56.99 & 59.96 & 21.35 \\
\hline$T_{6}$ & Hexaconazole & 62.68 & 59.46 & 57.28 & 57.71 & 57.06 & 58.84 & 9.85 \\
\hline $\mathbf{T}_{7}$ & $\begin{array}{l}\text { Tebuconazole+ } \\
\text { Trifloxystrobin }\end{array}$ & 64.18 & 61.13 & 60.07 & 60.22 & 60.13 & 61.15 & 6.74 \\
\hline \multirow[t]{2}{*}{$\mathbf{T}_{8}$} & Control (Water spray) & 64.39 & 58.15 & 53.27 & 49.26 & 45.56 & 54.13 & 41.33 \\
\hline & $\mathrm{CD}(\mathrm{P}=0.05)$ & NS & 0.086 & 0.236 & 0.924 & 1.005 & & \\
\hline
\end{tabular}

DAS - Days after second spraying

*Mean of three replications

Table.7 Cost benefit ratio of the treatments on scented rose

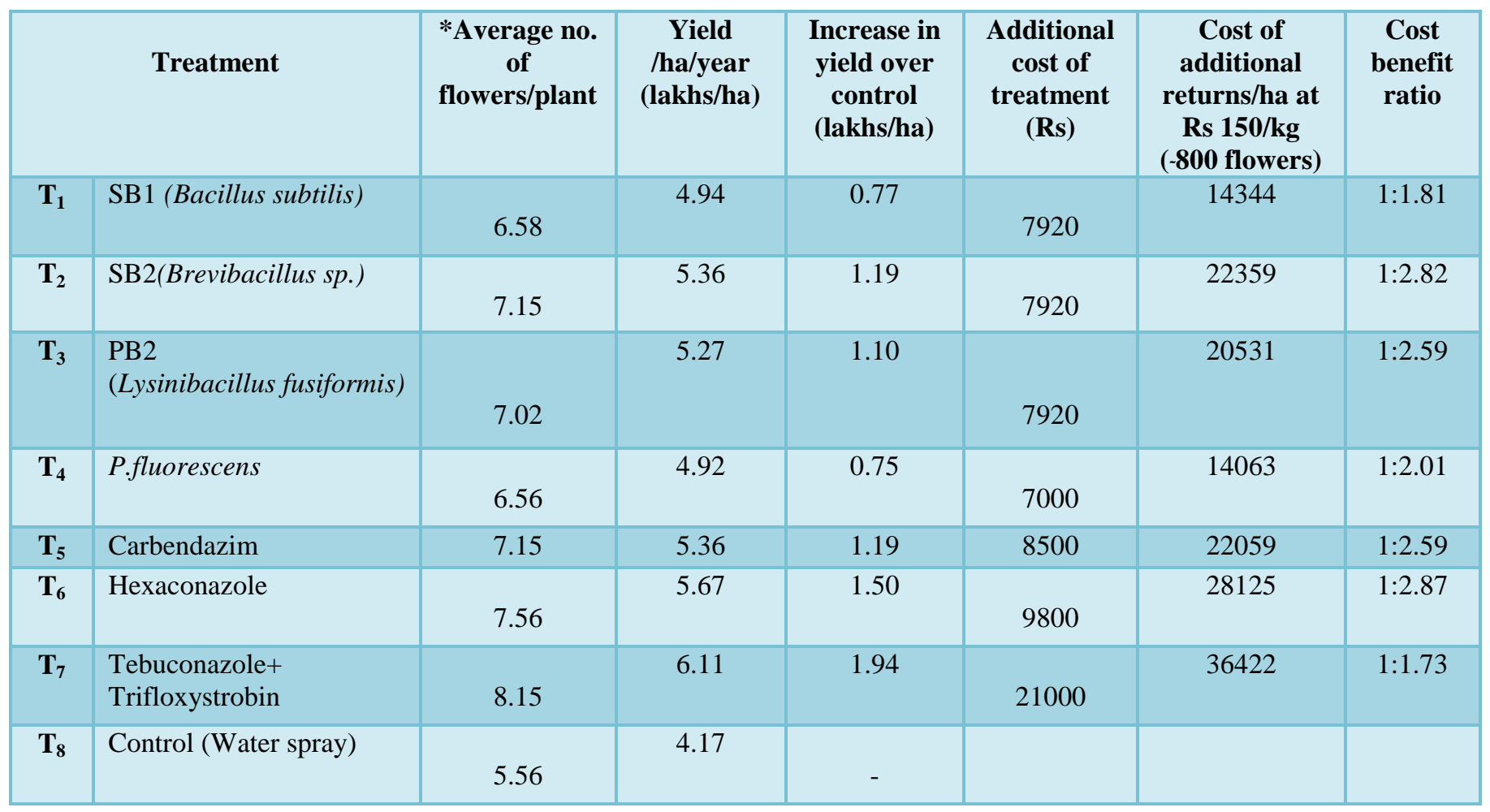



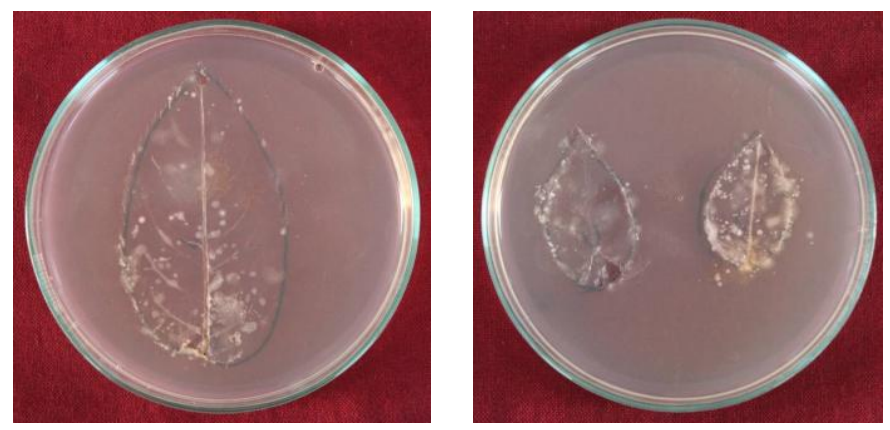

Fig.1 Isolation of phylloplane bacteria by leaf impression method

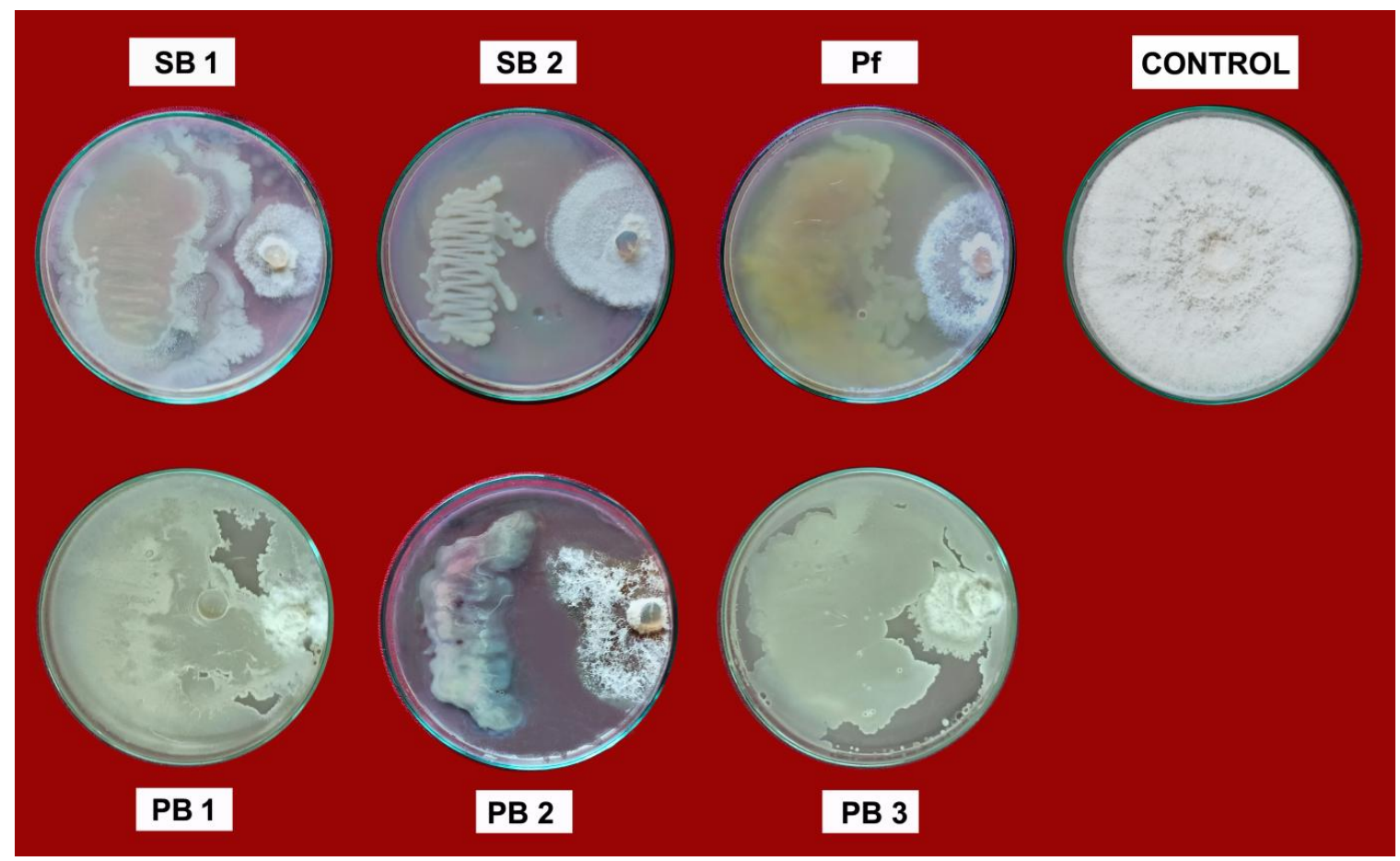

Fig.2 Effect of phylloplane and soil bacteria on the mycelial growth of D. rosae in vitro *SB-Soil bacteria; PB-Phylloplane bacteria

Yasin and Ahmed (2016) reported that among the 16 rhizobacteria isolated from the rhizosphere soil (collected from rhizosphere region of the healthy rose plants), two strains RB4 (Pseudomonas fluorescens) and RB11 (B. subtilis) controlled the black spot disease of rose by triggering the accumulation of elevated quantity of peroxidises, phenolics, polyphenol oxidase, phenylalanine ammonialyase, ascorbic acid and total soluble protein. Karthikeyan et al., (2007) tested eight antagonistic microbes against black spot pathogen in rose under in vivo condition and reported that two antagonist Trichoderma viride and Pseudomonas fluorescens Pf 1 inhibited the mycelial growth of pathogen by stimulation of synthesis of defense related enzymes in host leaves.

The present study imparted that black spot disease of rose caused by Diplocarpon rosae can be controlled by antifungal activity of new strains of Brevibacillus sp. (SB2) and Lysinibacillus fusiformis (PB2) as biocontrol agents which were isolated and identified from rhizosphere and phylloplane region of 
the rose plant respectively. The antimicrobial secondary metabolites produced by these bacteria could be identified and produced in mass quantity to be used against the disease. The secondary metabolites can be used as an effective and eco-friendly alternative to chemical fungicides.

\section{References}

Bhaskaran, R, D Purushothaman, and K Ranganathan. 1974. "Physiological changes in rose leaves infected by Diplocarpon rosae." Journal of Phytopathology, 79 (3): 231-236.

Bordi, Christophe, and Sophie de Bentzmann. 2011. "Hacking into bacterial biofilms: a new therapeutic challenge." Annals of intensive care, 1 (1): 19.

Bowen, KL, and RS Roark. 2001. "Management of black spot of rose with winter fungicide treatment." Plant disease, 85 (4): 393-398.

Debener, T, R Drewes-Alvarez, and K Rockstroh. 1998. Identification of five physiological races of blackspot, Diplocarpon rosae, Wolf on roses. Plant breeding, 117 (3): 267-270.

Endimiani, Andrea, Francesco Luzzaro, Beatrice Pini, Gianfranco Amicosante, Gian Maria Rossolini, and Antonio Q Toniolo. 2006. "Pseudomonas aeruginosa bloodstream infections: risk factors and treatment outcome related to expression of the PER-1 extended-spectrum beta-lactamase." $B M C$ infectious diseases, 6 (1): 52.

Gachomo, Emma W, Manfredo J Seufferheld, and Simeon O Kotchoni. 2010. "Melanization of appressoria is critical for the pathogenicity of Diplocarpon rosae." Molecular biology reports, 37 (7): 35833591.
Gomes, LH, KMR Duarte, FG Andrino and FCA Tavares. 2000. "A simple method for DNA isolation from Xanthomonas spp." Scientia Agricola, 57: 553-555.

Karthikeyan, Muthusamy, Ramanujam Bhaskaran, Subramanian Mathiyazhagan, and Rethinasamy Velazhahan. 2007. "Influence of phylloplane colonizing biocontrol agentson the black spot of rose caused by Diplocarpon rosae." Journal of Plant Interactions, 2 (4): 225-231.

Kunert, Anja, Josephine Losse, Christin Gruszin, Michael Hühn, Kerstin Kaendler, Stefan Mikkat, Daniela Volke, Ralf Hoffmann, T Sakari Jokiranta, and Harald Seeberger. 2007. "Immune evasion of the human pathogen Pseudomonas aeruginosa: elongation factor Tuf is a factor $\mathrm{H}$ and plasminogen binding protein." The Journal of Immunology, 179 (5): 2979-2988.

Nelson, Scot. 2012. "Black spot of rose in Hawaii." Plant disease, 80: 1-6.

Talbot, George H, John Bradley, John E Edwards Jr, David Gilbert, Michael Scheld, and John G Bartlett. 2006. "Bad bugs need drugs: an update on the development pipeline from the Antimicrobial Availability Task Force of the Infectious Diseases Society of America." Clinical infectious diseases, 42 (5): 657-668.

Wenefrida, I, and JA Spencer. 1993. "Marssonina rosae variants in Mississippi and their virulence on selected rose cultivars." Plant disease (USA), 77 (3): 246-248.

Yasin, NA, and S Ahmed. 2016. "Induction of defence-related biochemicals by rhizosphere bacteria against black spot disease of rose." Biological agriculture \& horticulture, 32 (1): 34-46.

\section{How to cite this article:}

Shalini, M. Jayasekhar, K. G. Sabarinathan, R. Akila and Kannan, R. 2020. Antifungal Activity of New Bacterial Biocontrol Agents against Diplocarpon rosae Causing Black Spot Disease of Rose. Int.J.Curr.Microbiol.App.Sci. 9(05): 3124-3133.

doi: https://doi.org/10.20546/ijcmas.2020.905.370 